\title{
Analysis and Design of LDPC Codes for Time-Selective Complex-Fading Channels
}

\author{
Kaiann Fu, Student Member, IEEE, and Achilleas Anastasopoulos, Member, IEEE
}

\begin{abstract}
Pilot-symbol-assisted (PSA) low-density parity-check (LDPC) codes are analyzed using density evolution techniques for a frequency-nonselective, time-selective fading channel where the fading affects both the amplitude and the phase of the transmitted signal. The performance of several message-passing estimation/decoding receivers is investigated, and the optimal energy allocation between pilot and coded symbols is evaluated. Several messagepassing estimation/decoding receivers are shown to fall under a single unifying description which leads to significant simplification of the analysis and design of the PSA LDPC codes and receivers. The immediate surprising result is that for the described class of receivers, the best codes for the case of perfect channel state information (CSI) at the receiver are also the best codes when no CSI is available at the receiver, regardless of the channel dynamics. A class of more elaborate receivers that perform iterative joint decoding/estimation is also presented and it is shown that codes designed specifically for these receivers provide an additional performance gain.
\end{abstract}

Index Terms-Low-density parity-check (LDPC) codes, complex fading channels, pilot-symbol-assisted (PSA) codes, iterative decoding, density evolution.

\section{INTRODUCTION}

$\mathbf{L}$ OW-DENSITY PARITY-CHECK (LDPC) codes in conjunction with iterative decoding based on message-passing algorithms have been shown to achieve excellent performance over the AWGN channel [1], [2]. Their potential as capacityachieving codes for more realistic wireless channels has not been established yet. However, experimental evidence as well as some preliminary analytical results [3] have led to the conjecture [4] that LDPC - or in general turbo-like codes-can achieve capacity for a wide range of channels. ${ }^{1}$

Recently, it was demonstrated that LDPC codes show very good performance over the memoryless frequency-nonselective (i.e., flat) Rayleigh fading channel [3] and for the noncoherent additive white Gaussian noise AWGN channel [5], [6]. A more realistic channel is considered in this paper. Specifically, a frequency-nonselective, time-selective complex fading channel is

Manuscript received July 7, 2003; revised January 16, 2004 and April 3, 2004; accepted April 9, 2004. The editor coordinating the review of this paper and approving it for publication is G. Vitetta. The work of K. Fu was supported in part by a National Science Foundation Graduate Fellowship. This work was supported in part by National Science Foundation Grant CCR-0219531. This paper was presented in part at the IEEE Globecom Communications Conference, Taipei, Taiwan, November 2002.

The authors are with the Electrical Engineering and Computer Science Department, University of Michigan, Ann Arbor, MI 48109-2122 USA (e-mail: kaiann@umich.edu; anastas@umich.edu).

Digital Object Identifier 10.1109/TWC.2005.846985

${ }^{1}$ Although the focus of this paper is on LDPC codes, the decoding algorithms and analysis techniques can also be applied to other turbo-like codes. considered, where both the effects of amplitude and phase variation are taken into account. This is certainly a more realistic model than the one assuming only amplitude [3] or only phase [5], [6] variations of the transmitted signal. Furthermore, in this work, the memoryless assumption of [3] is raised and channel dynamics are explicitly taken into account by considering a block-independent fading model. In particular, the complex fading is considered constant for a block of length $N$ (which can be thought of as the channel coherence time) and independent from block to block. This model is quite accurate for frequency-hopping or time-division multiple-access schemes. It is also a good model for more general channels since it simplifies analysis, while modeling the dynamics of the fading process through a single parameter $N$.

Although coding for this channel is generally complicated as evidenced in [7], ${ }^{2}$ in this paper we consider a simple coding scheme, namely pilot-symbol-assisted (PSA) LDPC codes. ${ }^{3}$ Pilot symbol(s) of specified energy are added in the beginning of each block of length $N$ to establish a reference for the phase of the symbols and to aid (implicitly) the estimation/decoding process. We consider a general scenario where both the number of pilots as well as the pilot energy are design parameters. ${ }^{4}$ Clearly, the quality of channel estimation improves with increased energy in the pilots, while the quality of the decoded symbols depends on the energy spent on the coded symbols. Thus, for a fixed energy per information bit, a tradeoff between allocation of energy to the pilots and coded symbols exists. Using density evolution [1], [2], this tradeoff is studied without resorting to simulations, and the optimal power allocation is obtained for several PSA receiver structures first suggested and analyzed in [6] for the block-independent noncoherent AWGN channel. The optimized PSA LDPC codes are shown to have significantly improved performance over the nonoptimized codes, and the optimal energy allocation to pilots and coded symbols depends both on the channel coherence time and the particular receiver used. Thus, a quantitative answer, which is closely related to a particularly simple family of codes and decoders, is obtained to the question of "how much pilot energy is

\footnotetext{
${ }^{2}$ It is noted that the work in [7] refers to the more interesting case of multipleinput/multiple-output complex fading channels.

${ }^{3}$ When the channel state information (CSI) is known perfectly at the receiver (i.e., for the perfect CSI receiver), the PSA scheme is not needed and, hence, it is not used.

${ }^{4}$ One can consider an equivalent-or actually a more bandwidth efficient-system having a single pilot per block of length $N$ with a specified energy. However, multiple pilots per block are useful when the total pilot energy needs to be spread in time due to amplifier dynamic range constraints at the transmitter.
} 
required for transmission in the noncoherent fading channel?" [8], [9] for the single-antenna transmission scenario.

In this paper, two classes of receivers are considered. In the first one, channel estimation is performed once, followed by iterative decoding. In the second one, estimation and decoding are performed in an iterative fashion thus resulting in performance closer to that of belief propagation (i.e., the sum-product algorithm), but with only a fraction of its complexity. As mentioned earlier, these receiver structures have been discussed before in the context of LDPC decoding in the noncoherent AWGN channel [6], and similar receivers have been proposed also in the context of decoding turbo codes in the presence of time-selective fading [10], [11]. The presented formulation, however, leads to a number of surprising results regarding the analysis and design of these codes.

First, it is shown that for a given (regular or irregular) LDPC code, and for the first class of receivers described above, performance analysis in the form of density evolution only needs to be performed once regardless of the channel dynamics $N$, the number of pilots, and the energy allocation to pilots and coded symbols. Moreover, this analysis is exactly the same as if the code operated in an equivalent fading channel with perfect channel-state information (CSI) available at the receiver and with a smaller signal-to-noise ratio.

Second, design and optimization of irregular LDPC codes for the first class of receivers discussed above is greatly simplified. In particular, the design process can be decomposed into two steps. The first one, which is the most time consuming, involves degree polynomial optimization and is usually performed by computer search using differential evolution techniques [3], [12]. It is shown here that this step need only be performed once regardless of whether the code operates with perfect CSI or with no CSI at the receiver and regardless of the channel dynamics, $N$. Thus, the best codes for perfect CSI are also the best codes for no CSI and for any channel dynamics, for the receivers of the first class. The second step involves optimization of the allocation of energy to the pilots and coded symbols and can be performed using closed-form expressions.

The above two results are very different from the corresponding results observed in [5], [6] for the noncoherent AWGN channel. In particular, in the noncoherent AWGN channel, analysis and code design can not be unified as discussed above for the complex fading channel and must be conducted separately for each receiver, for each value of the channel coherence time $N$, and for each value of the energy allocation to pilots and coded symbols.

When additional complexity can be afforded, one can utilize the receiver structures of the second class that perform estimation and decoding in an iterative fashion. When these receivers are used, the two results mentioned above are no longer valid. We optimize PSA LDPC codes for these receivers and show performance gains over the receivers of the first class.

The rest of the paper is structured as follows. In Section II, we discuss the system and channel model under consideration. The specific message-passing estimation/decoding algorithms are described in Section III while performance analysis using density evolution and code design are presented in Section IV.

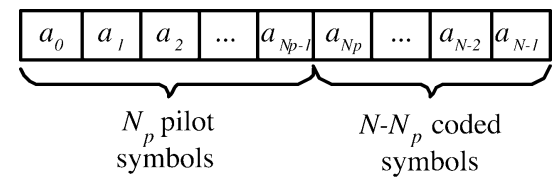

Fig. 1. Block diagram for the PSA scheme in a block of length $N$.

Section V presents numerical results followed by the conclusion in Section VI.

\section{System AND ChANNEl Model}

For the channel model, we consider a block-independent fading model where the fading is constant for a block of $N$ symbols and is independent from block to block. To facilitate channel estimation in each block, we use the PSA scheme depicted in Fig. 1 where the first $N_{p}$ transmitted symbols are pilot symbols each with energy $E_{p}$ followed by $N-N_{p}$ coded symbols each with energy $E_{s}$. For the block fading model, the exact placement of the pilot symbols in the block will not affect the performance.

An LDPC code is used as the underlying code for the system. For each block of length $N$, we transmit symbols $(-1)^{a_{k}}$ for all $k=0, \ldots, N-1$ with pilot symbols $a_{k}=0$ for $k=$ $0, \ldots, N_{p}-1$ and with coded symbols from the LDPC code $a_{k} \in\{0,1\}$ for $k=N_{p}, \ldots, N-1$. The analysis presented in this paper is applied to general irregular LDPC codes with maximum variable (check) node degree of $d_{v}\left(d_{c}\right)$ and degree polynomials $\lambda(x)$ and $\rho(x)$ as defined in [2], but they can also be applied to other codes which can be represented by a factor graph.

The received symbols for each block of length $N$ can be expressed as

$$
z_{k}=c \sqrt{E_{k}}(-1)^{a_{k}}+n_{k} \quad k=0, \ldots, N-1
$$

where

$$
E_{k}= \begin{cases}E_{p} & k=0, \ldots, N_{p}-1 \\ E_{s} & k=N_{p}, \ldots, N-1\end{cases}
$$

In the above equations, the fading coefficient $c$ is modeled as a zero-mean circular complex Gaussian random variable with $E\left[|c|^{2}\right]=1$. Thus, the fading amplitude has a Rayleigh density while the fading phase has a uniform density in $[0,2 \pi)$. The additive noise is modeled by independent zero-mean circular complex Gaussian random variables $n_{k}$ with $E\left[\left|n_{k}\right|^{2}\right]=N_{0}$.

The effective energy per information bit is

$$
E_{b}=\frac{1}{R}\left(E_{s}+\frac{N_{p} E_{p}}{N-N_{p}}\right)
$$

where $R$ is the rate of the LDPC code. Due to the pilot transmission, the overall rate (throughput) of the code is reduced to $R_{c}=R\left(N-N_{p}\right) / N$ (bits/complex dimension). For the channel model presented above, if no constraints on the pilot energy $E_{p}$ exist, then a PSA scheme with $N_{p}>1$ is suboptimal since we can simply put the total energy $N_{p} E_{p}$ into a single pilot and increase the total throughput by a factor of $(N-1) /\left(N-N_{p}\right)$. However, if the peak power is limited due to linearity constraints at the transmitter amplifier, then multiple pilots per block might 


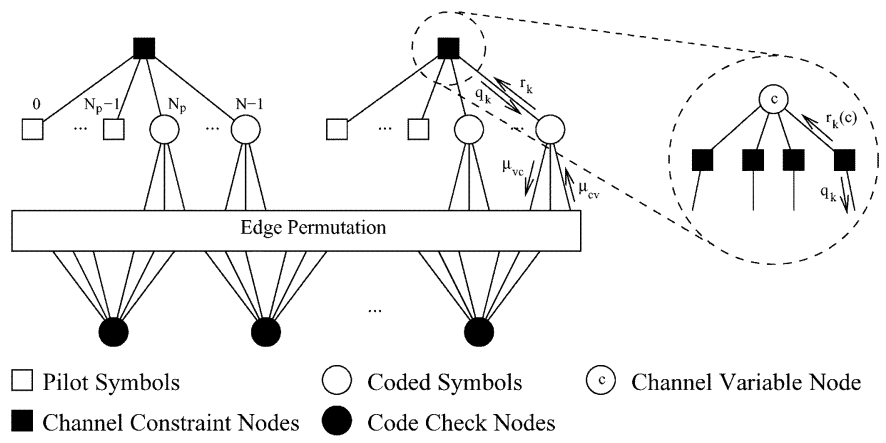

(a)

(b)

Fig. 2. (a) Factor graph for a PSA LDPC code in a flat, block-independent fading channel. (b) Equivalent representation of the channel constraint node in (a). The channel variable node represents the fading coefficient $c$.

be necessary to achieve higher effective pilot energies at the cost of lowering the overall throughput.

We consider a regular PSA scheme where the number of pilot symbols per block $N_{p}$ and the energy per pilot symbol $E_{p}$ are the same for all blocks. The factor graph [13] of this system is shown in Fig. 2(a). Each transmitted symbol, i.e., each pilot symbol and each coded symbol, is represented by a variable node while each parity check is represented by a code check node. In addition, the variable nodes are connected to channel constraint nodes which represent the constraints imposed by the fading channel. In an equivalent representation, each channel constraint node can be decomposed as in Fig. 2(b) to explicitly express the dependence on the fading coefficient $c$. In this case, a variable node describes $c$ while each of the channel constraint nodes here represents the channel constraint, based on $c$, on a single transmitted symbol. It will be shown in Section III that the two factor graph representations are indeed equivalent. Although a channel interleaver is usually inserted between the encoder and the channel, this device is not necessary for LDPC codes due to the fact that interleaving is inherent since the order of the coded bits is irrelevant.

\section{DeCODING AlgorithmS}

The PSA LDPC codes can be iteratively decoded by messagepassing algorithms operating on the factor graph of the system. Since message exchange between variable and check nodes for LDPC codes is well understood, we concentrate on the messages generated at the channel constraint node in Fig. 2(a). In the following, we describe several receiver algorithms which rely on different options for message generation at the channel constraint node. The receivers discussed here were proposed in [6] for the noncoherent AWGN channel. Thus in the following we concentrate on the details of these receivers that are specific to the channel under consideration. In particular, 1) analytical results are easier to obtain here compared to [6] since closed-form expressions exist for the message densities as will be shown in Section IV, and 2) analysis and code design for different system and channel parameters are unified for the first class of receivers in the complex fading channel considered here, which was not the case for the noncoherent AWGN channel in [6].
1) Perfect CSI: When perfect CSI is available at the receiver, i.e., when the fading amplitude $c$ is exactly known at the receiver side, the message $q_{k}$ from the channel constraint node to the $k$ th variable node can be evaluated as in [3]

$$
q_{k}=\log \frac{f\left(z_{k} \mid a_{k}=0, c\right)}{f\left(z_{k} \mid a_{k}=1, c\right)}=\frac{4 \sqrt{E_{s}}}{N_{0}} \Re\left\{z_{k} c^{*}\right\}
$$

where $\Re(x)$ denotes the real part of $x$. Since the fading coefficient is known, no pilot symbol is necessary. In addition, since $q_{k}$ is independent of all incoming messages $r_{i}$ [as shown in Fig. 2(a)] for $i \in\{0, \ldots, N-1\} \backslash\{k\}$, the message $q_{k}$ is evaluated once at the beginning of the iterative algorithm and does not change in subsequent iterations.

2) Sum-Product Algorithm: When CSI is not available at the receiver, iterative detection and estimation can be performed using the sum-product algorithm. In particular, the channel constraint node generates a log-likelihood ratio for the $k$ th variable node based on the information it receives from the $N-1$ other variable nodes and on the $N$ channel output values. Assuming that the incoming message from the $i$ th variable node to the channel constraint node is a log-likelihood ratio of the form $r_{i}=\log \left(p_{i}\left(a_{i}=0\right) / p_{i}\left(a_{i}=1\right)\right),{ }^{5}$ the outgoing message to the $k$ th variable node can be expressed as

$$
\begin{aligned}
q_{k} & =\log \frac{f\left(\mathbf{z} \mid a_{k}=0\right)}{f\left(\mathbf{z} \mid a_{k}=1\right)} \\
& =\log \frac{\sum_{\mathbf{a}: a_{k}=0} \mathcal{C N}_{N}(\mathbf{z} ; \mathbf{0}, \mathbf{K}(\mathbf{a})) \prod_{i=0, i \neq k}^{N-1} p_{i}\left(a_{i}\right)}{\sum_{\mathbf{a}: a_{k}=1} \mathcal{C N}_{N}(\mathbf{z} ; \mathbf{0}, \mathbf{K}(\mathbf{a})) \prod_{i=0, i \neq k}^{N-1} p_{i}\left(a_{i}\right)}
\end{aligned}
$$

where $\mathbf{z}=\left(z_{0}, \ldots, z_{N-1}\right)^{T}$, the summations are over all possible vectors $\mathbf{a} \in\{0,1\}^{N}$ with the $k$ th element given as $a_{k}=0$ or $a_{k}=1$, and $\mathcal{C} \mathcal{N}_{N}(\mathbf{z} ; \mathbf{0}, K(\mathbf{a}))$ denotes an $N$-dimensional zero-mean complex Gaussian probability density function with covariance matrix

$$
\mathbf{K}(\mathbf{a})=E\left[\mathbf{z z}^{H} \mid \mathbf{a}\right]=\boldsymbol{\mu} \boldsymbol{\mu}^{H}+N_{0} \mathbf{I}_{N}
$$

where $\boldsymbol{\mu}=\left(\mu_{0}, \ldots, \mu_{N-1}\right)^{T}, \mu_{i}=\sqrt{E_{i}}(-1)^{a_{i}}$ for all $i \in$ $\{0, \ldots, N-1\}$, and $\mathbf{I}_{N}$ is the $N \times N$ identity matrix.

The expression in (5) does not explicitly involve the unknown channel parameter $c$ since it has been implicitly integrated out of the above expression. The message $q_{k}$ can also be expressed following the equivalent factor graph representation in Fig. 2(b) where the dependence on the complex fading coefficient $c$ is explicit,

$$
\begin{aligned}
q_{k}=\log \frac{\int_{\mathbb{C}} f\left(\mathbf{z} \mid a_{k}=0, c\right) f(c) d c}{\int_{\mathbb{C}} f\left(\mathbf{z} \mid a_{k}=1, c\right) f(c) d c} \\
=\log \frac{\int_{\mathbb{C}} \mathcal{C N}\left(z_{k} ; \sqrt{E_{s}} c, N_{0}\right) \mathcal{C N}(c ; 0,1) \prod_{i=0, i \neq k}^{N-1} r_{i}(c) d c}{\int_{\mathbb{C}} \mathcal{C N}\left(z_{k} ;-\sqrt{E_{s}} c, N_{0}\right) \mathcal{C N}(c ; 0,1) \prod_{i=0, i \neq k}^{N-1} r_{i}(c) d c}
\end{aligned}
$$

\footnotetext{
${ }^{5}$ It is assumed that the incoming messages corresponding to the pilot symbols are $r_{k}=+\infty$ for $k=0, \ldots, N_{p}-1$.
} 
where $\mathbb{C}$ is the complex plane and

$$
r_{i}(c)=\sum_{a=0}^{1} \mathcal{C N}\left(z_{i} ; \sqrt{E_{s}} c(-1)^{a} ; N_{0}\right) p_{i}\left(a_{k}=a\right)
$$

for $i=0, \ldots, N-1$. By explicitly integrating over $c$, it can be shown that the messages in (5) and (7) are equal, thus establishing the equivalence between the factor graphs in Fig. 2(a) and (b).

It is a well-known fact that if the factor graph is cycle-free, then at the termination of the sum-product algorithm, the maximum a posteriori (MAP) estimate of each coded symbol is obtained [13]. However, evaluating the channel-to-variable-node message in (5) has exponential complexity in $N$. Similarly, evaluating the complex integrals in (7) has-at least theoretically-infinite complexity. The integration can be approximated by quantizing the amplitude and phase of $c$, which is usually what is done in practice [14]. Motivated by the high complexity of the exact sum-product algorithm in evaluating the channel-to-variable-node message, suboptimal implementations of this operation are suggested in the next paragraphs 3 ) and 5). In addition, the hypothetical receiver described in paragraph 4) serves as an upper bound on the performance of the exact sum-product algorithm.

3) Pilot Only Detection: In the pilot only (PO) receiver, only the pilot symbol(s) are used by the channel constraint node to obtain information about the channel. The message $q_{k}$ for the $k$ th variable node is computed by

$$
\begin{aligned}
q_{k} & =\log \frac{f\left(z_{k}, z_{0}, \ldots, z_{N_{p}-1} \mid a_{k}=0\right)}{f\left(z_{k}, z_{0}, \ldots, z_{N_{p}-1} \mid a_{k}=1\right)} \\
& =\frac{4 \sqrt{E_{s}} \Re\left\{z_{k} \sqrt{E_{p}} \sum_{i=0}^{N_{p}-1} z_{i}^{*}\right\}}{N_{0}\left(N_{0}+N_{p} E_{p}+E_{s}\right)} .
\end{aligned}
$$

Similar to the perfect CSI case, the message $q_{k}$ is independent of all messages $r_{i}$ for $i \in\{0, \ldots, N-1\} \backslash\{k\}$, so it is evaluated once at the beginning of the iterative process and remains the same at each iteration.

4) Pilot and Data Correct Decision Feedback: The pilot and data correct decision feedback (PDCDF) receiver is a hypothetical receiver which cannot be implemented in practice. However, it can be used to derive a lower bound on the minimum $E_{b} / N_{0}$ required to achieve error-free transmission using the exact sum-product algorithm. In the PDCDF receiver, the output of the channel constraint node for the $k$ th symbol is determined by the $N_{p}$ pilot symbols as well as the other $N-N_{p}-1$ coded symbols in the block. It is assumed that due to the presence of a genie, the correct values of these $N-N_{p}-1$ coded symbols $\mathbf{a}_{k}^{\prime}=\left(a_{N_{p}}, \ldots, a_{k-1}, a_{k+1}, \ldots, a_{N-1}\right)$ are available in each iteration. The resulting message is of the form shown in (10) located at the bottom of the page.
Once again, it is observed that the messages $q_{k}$ remain the same at each iteration. In addition, (10) is equivalent to (9) with $\left(N-N_{p}-1\right)$ additional pilot symbols, each with energy $E_{s}$. Thus, as will be verified in the next section, the PDCDF receiver is equivalent to the $\mathrm{PO}$ receiver where the effective pilot energy is $N_{p} E_{p}+\left(N-N_{p}-1\right) E_{s}$, since the other $N-N_{p}-1$ coded symbols in the block also act as pilots for the $k$ th variable node. Following an argument similar to the one used in [6], one can show rigorously that the PDCDF receiver cannot perform worse than the sum-product algorithm.

5) Quantized Decision Feedback: In the PDCDF receiver, $N-N_{p}-1$ coded symbols in a block act as pilots for calculating the log-likelihood ratio for the $k$ th variable node. This is a hypothetical scenario where the receiver knows exactly the values of these $N-N_{p}-1$ symbols. However, when the messages $r_{i}$ entering the channel constraint node are strongly biased toward $\pm \infty$, the corresponding symbols can act as pilots as well. Motivated by this observation, we propose an ad-hoc algorithm, the quantized decision feedback (QDF) receiver, which operates as follows. The incoming messages are first quantized according to the following rule:

$$
\hat{r}_{i}= \begin{cases}+\infty, & \text { if } r_{i}>T \\ 0, & \text { if }-T \leq r_{i} \leq T \\ -\infty, & \text { if } r_{i}<-T\end{cases}
$$

for $i \in\{0, \ldots, N-1\} \backslash\{k\}$ where $T$ is a predetermined threshold value. Using these quantized messages $\hat{r}_{i}$ in evaluating the message $q_{k}$ is equivalent to assuming that symbols for which $\left|r_{i}\right|>T$ act as pilots, while those for which $\left|r_{i}\right| \leq T$ do not contribute to the estimation process. For notational simplicity, let $\hat{s}_{i}=\operatorname{sgn}\left(\hat{r}_{i}\right)$ where $\operatorname{sgn}(x)$ equals +1 if $x>0,0$ if $x=0$, and -1 if $x<0$. When $\hat{s}_{i} \neq 0, \hat{s}_{i}$ represents an estimate of the transmitted symbol $(-1)^{a_{i}}$. Also, let $\mathbf{z}_{k}^{\prime}$ and $\hat{\mathbf{r}}_{k}^{\prime}$ be the vector of $z_{i}$ 's and $\hat{r}_{i}$ 's, respectively, for all indices $i$ such that $i \in\{0, \ldots, N-1\}, i \neq k$, and $\hat{r}_{i} \neq 0$. The resulting expression for the message $q_{k}$ is (12),

$$
\begin{aligned}
q_{k} & =\log \frac{f\left(\mathbf{z}_{k}^{\prime} \mid \hat{\mathbf{r}}_{k}^{\prime}, a_{k}=0\right)}{f\left(\mathbf{z}_{k}^{\prime} \mid \hat{\mathbf{r}}_{k}^{\prime}, a_{k}=1\right)} \\
& =\frac{4 \sqrt{E_{s}} \Re\left\{z_{k}\left(\sqrt{E_{p}} \sum_{i=0}^{N_{p}-1} z_{i}+\sqrt{E_{s}} \sum_{i=N_{p}, i \neq k}^{N-1} \hat{s}_{i} z_{i}\right)^{*}\right\}}{N_{0}\left(N_{0}+N_{p} E_{p}+N_{k}^{\prime} E_{s}+E_{s}\right)}
\end{aligned}
$$

where $N_{k}^{\prime}=\sum_{i=N_{p}, i \neq k}^{N-1}\left|\hat{s}_{i}\right|$ is the number of nonzero quantized messages excluding messages from pilot symbols and the one corresponding to the $k$ th variable node. It is emphasized

$$
\begin{aligned}
q_{k} & =\log \frac{f\left(\mathbf{z} \mid \mathbf{a}_{k}^{\prime}, a_{k}=0\right)}{f\left(\mathbf{z} \mid \mathbf{a}_{k}^{\prime}, a_{k}=1\right)} \\
& =\frac{4 \sqrt{E_{s}} \Re\left\{z_{k}\left(\sqrt{E_{p}} \sum_{i=0}^{N_{p}-1} z_{i}+\sqrt{E_{s}} \sum_{i=N_{p}, i \neq k}^{N-1}(-1)^{a_{i}} z_{i}\right)^{*}\right\}}{N_{0}\left(N_{0}+N_{p} E_{p}+E_{s}\left(N-N_{p}-1\right)+E_{s}\right)}
\end{aligned}
$$


that unlike the previous cases, in the QDF scheme, the channel constraint node must recalculate the message $q_{k}$ at each iteration, thus resulting in an iterative joint detection/estimation technique.

\section{Performance Analysis and Code Design}

The performance of the decoding algorithms in Section III is analyzed using density evolution, which involves evaluating the probability density functions (pdfs) of the messages exchanged between the nodes of the factor graph [1], given the pdfs of the initial messages. To apply density evolution, the all-zero codeword is assumed to have been transmitted. This assumption is not restrictive since the fading channel considered herein satisfies the channel symmetry condition $f\left(z_{k} \mid a_{k}=0\right)=f\left(-z_{k} \mid a_{k}=1\right)$. In addition, for these decoding algorithms, the channel constraint node preserves symmetry since a flip in sign of $z_{k}$ results in a flip in sign of $q_{k}$ at each iteration.

Under the standard assumptions of large girth (compared to the iteration number), the neighborhood of a graph is essentially a tree. In this case, all messages passed in the factor graph are independent and all calculated pdfs are exact. In the practical situation where cycles are present in the graph, it was shown in [1] that the average behavior of the code converges to the cycle-free case as the length of the code increases.

Using these analysis techniques, codes can be designed by optimizing irregular LDPC code degree polynomials and energy distributions $E_{p} / E_{s}$ to minimize the $E_{b} / N_{0}$ threshold, the value of $E_{b} / N_{0}$ required to achieve arbitrarily small error probability. In practical numerical optimization schemes, the optimization is completed subject to an upper bound on the maximum degrees $d_{v}$ and $d_{c}$ of the variable and check degree polynomials, respectively.

In this section, performance analysis and code design will be discussed for two sets of receivers: a) the perfect CSI, PO, and PDCDF receivers which belong to the class of receivers where channel estimation is only performed once and b) the QDF receiver which belongs to the class of receivers where channel estimation and decoding are performed in an iterative fashion.

\section{A. Perfect CSI, PO, and PDCDF Receivers}

For all the receivers described in Section III except the QDF receiver, the messages from the channel constraint nodes do not change with iterations. Thus, in order to perform density evolution for these receivers, it suffices to evaluate the initial message densities and then follow the standard pdf transformations described in [1] to trace the pdfs of the messages exchanged in the code portion of the factor graph. Furthermore, it can be observed that all $q_{k}$ messages described previously are of the form $q=C \Re\left\{x y^{*}\right\}$ where $C$ is a constant, $x$ and $y$ are zero-mean complex Gaussian variables, and the subscript $k$ is dropped for notational simplicity. The pdf of $q$ can be expressed as [15, Appendix B]

$$
f(q)=\frac{v_{1} v_{2}}{v_{1}+v_{2}}\left[e^{v_{2} q} u(-q)+e^{-v_{1} q} u(q)\right]
$$

where $u(q)$ is the unit step function, and $v_{1}$ and $v_{2}$ are given by

$$
\begin{aligned}
& v_{1}=\frac{2}{C\left(\sqrt{E\left[x x^{*}\right] E\left[y y^{*}\right]}+E\left[x y^{*}\right]\right)} \\
& v_{2}=\frac{2}{C\left(\sqrt{E\left[x x^{*}\right] E\left[y y^{*}\right]}-E\left[x y^{*}\right]\right)} .
\end{aligned}
$$

For the perfect CSI, PO, and PDCDF receivers, $v_{2}=v_{1}+1$ and thus, the initial pdfs can all be expressed with the same equation dependent on a single parameter $b$

$$
f(q)=\frac{b(1+b)}{1+2 b}\left[e^{(1+b) q} u(-q)+e^{-b q} u(q)\right]
$$

where $b=v_{1}$. For the perfect CSI receiver, $C=$ $4 \sqrt{E_{s}} / N_{0}, x=z_{k}, y=c, E\left[x x^{*}\right]=E_{s}+N_{0}, E\left[y y^{*}\right]=1$, and $E\left[x y^{*}\right]=\sqrt{E_{s}}$ which results in the following simplified expression for $b$ :

$$
b=\frac{1}{2} \sqrt{1+\frac{N_{0}}{E_{s}}}-\frac{1}{2} .
$$

Since no pilots are necessary, $b$ and $f(q)$ are independent of $N_{p}$ and $E_{p}$. For the PO receiver, $C=4 \sqrt{E_{s}} /\left(N_{0}\left(N_{0}+\right.\right.$ $\left.\left.N_{p} E_{p}+E_{s}\right)\right), x=z_{k}, y=\sqrt{E_{p}} \sum_{i=0}^{N_{p}-1} z_{i}, E\left[x x^{*}\right]=E_{s}+$ $N_{0}, E\left[y y^{*}\right]=N_{p} E_{p}\left(N_{p} E_{p}+N_{0}\right)$, and $E\left[x y^{*}\right]=N_{p} E_{p} \sqrt{E_{s}}$ which results in the following simplified expression for $b$ :

$$
b=\frac{1}{2} \sqrt{\left(1+\frac{N_{0}}{E_{s}}\right)\left(1+\frac{E_{s}}{E_{p, \text { eff }}} \frac{N_{0}}{E_{s}}\right)}-\frac{1}{2}
$$

where $E_{p, \text { eff }}=N_{p} E_{p}$. Clearly the performance of this receiver is only dependent on $E_{s} / N_{0}$ and $E_{p \text {,eff }} / E_{s}$. For the PDCDF receiver, $b$ is given by (17) with $E_{p \text {,eff }}=N_{p} E_{p}+\left(N-N_{p}\right.$ 1) $E_{s}$.

In light of the fact that the performance of the perfect CSI, $\mathrm{PO}$, and PDCDF receivers depends on the system parameters (i.e., $N, N_{p}, E_{s} / N_{0}, E_{p} / E_{s}$ ) only through a single parameter $b$, performance analysis using density evolution for a given LDPC code can be performed easily for all these receivers as follows.

First, using density evolution with initial message pdfs given in (15), the threshold value $b^{*}$ is obtained. The meaning of this value is that for any $b<b^{*}$ the iterative algorithm converges to zero probability of bit error as the number of iterations and the codelength increases, while for any $b>b^{*}$ the iterative decoding algorithm does not converge to zero probability of bit error. Implicit in the above argument is the fact that the performance of these receivers is monotonically decreasing with $b$. A proof of this monotonicity is provided in Appendix A. Since this step is independent of the particular receiver type and independent of the system parameters, this single number $b^{*}$ characterizes the performance of all these systems.

Once $b^{*}$ is found, the $E_{b} / N_{0}$ threshold can be obtained through closed-form expressions for each receiver type. To derive these closed-form expressions, we first find $\left(E_{b} / N_{0}\right)^{\prime}$, the minimum $E_{b} / N_{0}$ required to achieve a given value of $b$. For the perfect CSI receiver, one can solve (16) to obtain $\left(E_{s} / N_{0}\right)^{\prime}=(B-1)^{-1}$ where $B=(1+2 b)^{2}$, and, hence, obtain $\left(E_{b} / N_{0}\right)^{\prime}=\left(E_{s} / N_{0}\right)^{\prime} / R$. For the PO receiver, given system parameters $N, N_{p}$, and $R$, (17) and (3) involve the 
quantities $E_{s} / N_{0}, E_{p, \text { eff }} / E_{s}$, and $E_{b} / N_{0}$. Solving this system of two equations and three unknowns for $E_{b} / N_{0}$ by eliminating $E_{s} / N_{0}$, we obtain

$$
\frac{E_{b}}{N_{0}}=\frac{1}{R}\left(1+\frac{\rho}{N-N_{p}}\right) \frac{\rho+1+\sqrt{(\rho+1)^{2}+4(B-1) \rho}}{2(B-1) \rho}
$$

where $B=(1+2 b)^{2}$ and $\rho=E_{p \text {,eff }} / E_{s}$. The optimal $E_{p, \text { eff }} / E_{s}$ that minimizes $E_{b} / N_{0}$ is given by the following closed-form expression:

$$
\left(\frac{E_{p, \text { eff }}}{E_{s}}\right)^{\prime}=\frac{B\left(N-N_{p}\right)+\sqrt{B\left(N-N_{p}\right)}}{B+\sqrt{B\left(N-N_{p}\right)}} .
$$

This results in the minimum $E_{b} / N_{0}$ given by

$$
\begin{aligned}
\left(\frac{E_{b}}{N_{0}}\right)^{\prime}=\frac{1}{\mathrm{R}}\left[\left(\frac{N-N_{p}+1}{N-N_{p}}\right) \frac{1}{4 b(b+1)}\right. & \\
& \left.+\frac{1}{2 \sqrt{N-N_{p}}}\left(\frac{1}{b}+\frac{1}{b+1}\right)\right] .
\end{aligned}
$$

Similarly, for the PDCDF receiver when $N \geq N_{p}+2,{ }^{6}(17)$ and (3) give

$$
\frac{E_{b}}{N_{0}}=\frac{1}{R}\left(\frac{\rho+1}{N-N_{p}}\right) \frac{\rho+1+\sqrt{(\rho+1)^{2}+4(B-1) \rho}}{2(B-1) \rho} .
$$

The optimal $E_{p, \text { eff }} / E_{s}$ in this case satisfies the boundary condition

$$
\left(\frac{E_{p, \text { eff }}}{E_{s}}\right)^{\prime}=N-N_{p}-1
$$

which results in the minimum $E_{b} / N_{0}$ given by

$$
\begin{array}{r}
\left(\frac{E_{b}}{N_{0}}\right)^{\prime}=\frac{\sqrt{\left(N-N_{p}\right)^{2}+4(B-1)\left(N-N_{p}-1\right)}}{2 R(B-1)\left(N-N_{p}-1\right)} \\
+\frac{N-N_{p}}{2 R(B-1)\left(N-N_{p}-1\right)}
\end{array}
$$

For all the receivers here, $\left(E_{b} / N_{0}\right)^{\prime}$ is a monotonically decreasing function of $b$. Thus, since arbitrarily small bit error probability is possible if and only if $b<b^{*},\left(E_{b} / N_{0}\right)^{\prime}$ evaluated at $b=b^{*}$ is the $E_{b} / N_{0}$ threshold.

At this point a comparison with the analysis in [6] is in order. For the case of the noncoherent AWGN channel, finding the minimum $E_{b} / N_{0}$ value for the PO receiver required running density evolution for each value of $E_{s} / N_{0}$ and $E_{p \text {,eff }} / E_{s}$. Thus, searching over a two-dimensional space was required. However, in this work, due to the established equivalence among the first class of receivers, the search need only be conducted over a single parameter, $b$, to obtain analytical results for the perfect CSI, PO, and PDCDF receivers and for all system and channel parameters.

Code design is also unified for all three receivers and all system and channel parameters. In particular, code design can

\footnotetext{
${ }^{6}$ When $N=N_{p}+1$, the PDCDF receiver is a PO receiver and, hence, the equations in (19) apply in this case.
}

be divided into two steps. The first step relies on the fact that since the $E_{b} / N_{0}$ threshold is monotonically decreasing with $b^{*}$ as shown above, $b^{*}$ provides an ordering for LDPC codes for these receivers over the complex fading channel, i.e., LDPC codes with larger values of $b^{*}$ will have better performance. Thus, in the first step, the irregular LDPC degree polynomials are optimized using differential evolution [12], [3], subject to an upper bound on the maximum degrees, to produce an LDPC code with the largest $b^{*}$. The process of degree polynomial optimization is usually aided by imposing a constraint on the degree polynomials, known as the stability condition [2]. The stability condition for this channel is not presented here due to space limitations but was derived in [16]. In the case of perfect CSI receivers this step concludes the code design. For PO and PDCDF receivers, the second step is optimizing the energy distribution between pilots and coded symbols for the LDPC code found in the first step. This optimization can be determined through closed-form expressions as in (19) and (21).

The above discussion is essentially a constructive proof of the statement that the optimal LDPC codes for the perfect CSI receiver coincide with the optimal codes for the PO and PDCDF receivers for arbitrary channel dynamics $N$ and pilots per block $N_{p}$. More precisely, the optimal binary irregular LDPC code for all these cases should be the same. The only difference is the allocation of power to pilots and coded symbols which depends on the particular receiver used and the channel dynamics through the closed form expressions (19) and (21). It is emphasized that this equivalence results in a tremendous complexity reduction in designing good codes, since the degree optimization is usually the most time consuming part of the design process. Furthermore, even if design complexity is not an issue, the above statement guarantees that only a single binary LDPC code need be designed and utilized even if a system is supposed to operate in an environment where the channel dynamics are not known a priori. For instance, the LDPC codes optimized for the perfect CSI receiver in [3] are also optimal codes for the PO and PDCDF receivers for arbitrary dynamics (although this was not apparent to the authors of [3]).

\section{B. QDF Receiver}

Analysis for the QDF receiver is more complicated since the message pdfs at the $l$ th iteration from the channel constraint node are dependent on the pdfs $f^{(l-1)}\left(r_{i}\right)$ of the incoming messages $r_{i}$ for $i \in\{0, \ldots, N-1\}$ at the previous iteration. In the following, a brief description of the analysis at the channel constraint node is given (refer to [16] for more details). After quantizing the incoming messages, the probabilities of getting a correct and erroneous effective pilot are given by $p_{c}=P\left(\hat{r}_{i}=+\infty\right)=\int_{T}^{\infty} f^{(l-1)}\left(r_{i}\right) d r_{i}$ and $p_{e}=P\left(\hat{r}_{i}=-\infty\right)=\int_{-\infty}^{-T} f^{(l-1)}\left(r_{i}\right) d r_{i}$, respectively. The joint mass function $p^{(l-1)}(c, e)$ of correct and erroneous quantized signals at the $(l-1)$ st iteration is given by a multinomial distribution

$$
p^{(l-1)}(c, e)=\left(\begin{array}{c}
N-N_{p}-1 \\
c, e
\end{array}\right) p_{c}^{c} p_{e}^{e}\left(1-p_{c}-p_{e}\right)^{N-N_{p}-1-c-e}
$$

Since $c$ bits act as correct pilots, $e$ bits act as incorrect pilots, and $N-N_{p}-c-e-1$ bits do not contribute at all to the message 
evaluation, the outgoing message pdf $f(q \mid c, e)$ has the form of (13) with the constants $v_{1}$ and $v_{2}$ determined using (14) with

$$
\begin{aligned}
E\left[x x^{*}\right]= & E_{s}+N_{0} \\
E\left[y y^{*}\right]= & \left(N_{p} E_{p}+E_{s}(c-e)\right)^{2} \\
& +N_{0}\left(N_{p} E_{p}+E_{s}(c+e)\right) \\
E\left[x y^{*}\right]= & \sqrt{E_{s}}\left(N_{p} E_{p}+E_{s}(c-e)\right) .
\end{aligned}
$$

Finally, the message pdf from the channel constraint node at the $l$ th iteration can be evaluated using total probability as

$$
f^{(l)}(q)=\sum_{c=0}^{N-N_{p}-1} \sum_{e=0}^{N-N_{p}-1-c} f(q \mid c, e) p^{(l-1)}(c, e) .
$$

Unlike the simplified analysis in Section IV.A, the analysis here requires running separate density evolutions for each set of values of $E_{p} / E_{s}$ and $E_{s} / N_{0}$ in order to find the optimal set of values which minimizes the $E_{b} / N_{0}$ threshold for the given LDPC code, and this analysis must be completed separately for each set of values of $N$ and $N_{p}$.

Code design is also much more difficult for the QDF receiver and is dependent on the values of $N$ and $N_{p}$. The procedure for code design described here can in general be applied for any receiver type. Using differential evolution [3], [12], a search over degree polynomials, subject to an upper bound on the maximum degrees, can be completed to find the best LDPC code and energy distribution given $N$ and $N_{p}$. The performance of each LDPC code is determined in this process by using density evolution and searching over $E_{p} / E_{s}$ and $E_{s} / N_{0}$ to find the $E_{b} / N_{0}$ threshold.

Since this optimization process is computationally expensive, we simplify the process slightly by using the following ad-hoc iterative procedure. First, we initialize ${ }^{7} E_{p} / E_{s}=1$. A target value is set for $E_{b} / N_{0}$ and differential evolution is sused to find a code, with the given value of $E_{p} / E_{s}$, that can achieve arbitrarily small error probability for that value of $E_{b} / N_{0} . E_{b} / N_{0}$ is decreased until no such code can be found. With the best LDPC code found in the previous step, we then find the optimal $E_{p} / E_{s}$. The procedure is repeated for this new value of $E_{p} / E_{s}$. The iterative procedure ends when no improvement in $E_{b} / N_{0}$ is obtained from the last iteration. This ad-hoc procedure is not necessarily optimal and may not converge to the globally optimal solution. However, we repeated this process for several other initial $E_{p} / E_{s}$ values, and no significant improvement in performance was observed.

\section{NUMERICAL RESULTS}

In this section, density evolution results are generated using discretized density evolution [17]. In particular, each message is quantized using an 8-bit uniform quantizer with $2^{8}-1$ quantization levels. The range of the quantizer is roughly optimized to obtain low $E_{b} / N_{0}$ thresholds. Since messages are quantized, only probability mass functions need to be evaluated, thus the

\footnotetext{
${ }^{7}$ This initial value was chosen based on extensive experiments and our experience gained from [6], which showed that for LDPC degree polynomials with better performance, the optimal value of $E_{p} / E_{s}$ is close to 1 for the range of values of $N$ examined.
}

TABLE I

Rate 1/2 IRREgular LDPC DeGREe Polynomials Optimized FOR THE PERFECT CSI RECEIVER IN [3]

\begin{tabular}{cc|cc}
\hline \hline \multicolumn{2}{c|}{$\lambda(x)$} & \multicolumn{2}{|c}{$\rho(x)$} \\
\hline$\lambda_{1}$ & 0 & $\rho_{1}$ & 0 \\
$\lambda_{2}$ & 0.292439 & $\rho_{2}$ & 0 \\
$\lambda_{3}$ & 0.253636 & $\rho_{3}$ & 0 \\
$\lambda_{4}$ & 0.060454 & $\rho_{4}$ & 0 \\
$\lambda_{5}$ & 0 & $\rho_{5}$ & 0 \\
$\lambda_{6}$ & 0 & $\rho_{6}$ & 0.007254 \\
$\lambda_{7}$ & 0 & $\rho_{7}$ & 0.979220 \\
$\lambda_{8}$ & 0 & $\rho_{8}$ & 0.013526 \\
$\lambda_{9}$ & 0.031610 & & \\
$\lambda_{10}$ & 0.361861 & & \\
\hline \hline
\end{tabular}

resulting $E_{b} / N_{0}$ thresholds can be considered as upper bounds for belief propagation with continuous messages. However, discretized density evolution is exact for a practical receiver that quantizes the messages before iterative processing.

The results presented here for all receiver types use a single pilot symbol in each block, i.e., $N_{p}=1$, since this is the optimal choice for $N_{p}$. For the perfect CSI, PO, and PDCDF receivers, a search over $b$ with a resolution of 0.001 is conducted to find $b^{*}$, the largest $b$ such that the bit error rate is less than $10^{-8}$ in at most 1000 iterations. Results for the QDF receiver are obtained by searching over $E_{s} / N_{0}$ and $E_{p} / E_{s}$ values to find the minimum possible $E_{b} / N_{0}$ (with a resolution of $0.001 \mathrm{~dB}$ ) required to obtain a bit error rate less than $10^{-8}$ in at most 1000 iterations. For the QDF receiver, a separate search must be completed for each value of the channel coherence time $N$. At each iteration during discretized density evolution, the value of the threshold $T$ for the QDF receiver is numerically optimized to minimize the probability of bit error at that iteration.

For the regular $\left(d_{v}, d_{c}\right)=(3,6)$ LDPC code with a quantizer range of $(-35,35)$, discretized density evolution results in $b^{*}=0.203$. Using (16) and (17), this value of $b^{*}$ corresponds directly to the results obtained for the perfect CSI, PO, and PDCDF receivers in [16].

The irregular LDPC code described by the parameters given in Table $\mathrm{I}$ is a rate $1 / 2$ code, with maximum degrees $\left(d_{v}, d_{c}\right)=$ $(10,8)$, optimized for the perfect CSI receiver [3]. ${ }^{8}$ This code is also optimal for the $\mathrm{PO}$ and PDCDF receivers and for all values of the channel coherence time $N$, as shown in Section IV. Based on the results of [3], only minor performance improvement can be expected by using irregular LDPC codes with higher maximum degrees. For this code, discretized density evolution with a quantizer range of $(-17,17)$ results in $b^{*}=0.247$.

Fig. 3 displays a summary of the density evolution results for the irregular LDPC code. For the perfect CSI receiver, density evolution resulted in an $E_{b} / N_{0}$ threshold of $2.09 \mathrm{~dB}$, which agrees with the results in [3]. Similar to the regular $(3,6)$ LDPC

\footnotetext{
${ }^{8}$ Although the parameters $\lambda_{1}, \lambda_{5}, \lambda_{6}, \lambda_{7}, \lambda_{8}, \rho_{1}, \rho_{2}, \rho_{3}, \rho_{4}, \rho_{5}$ were set to zero in [3], optimization over these values showed no noticeable improvement.
} 


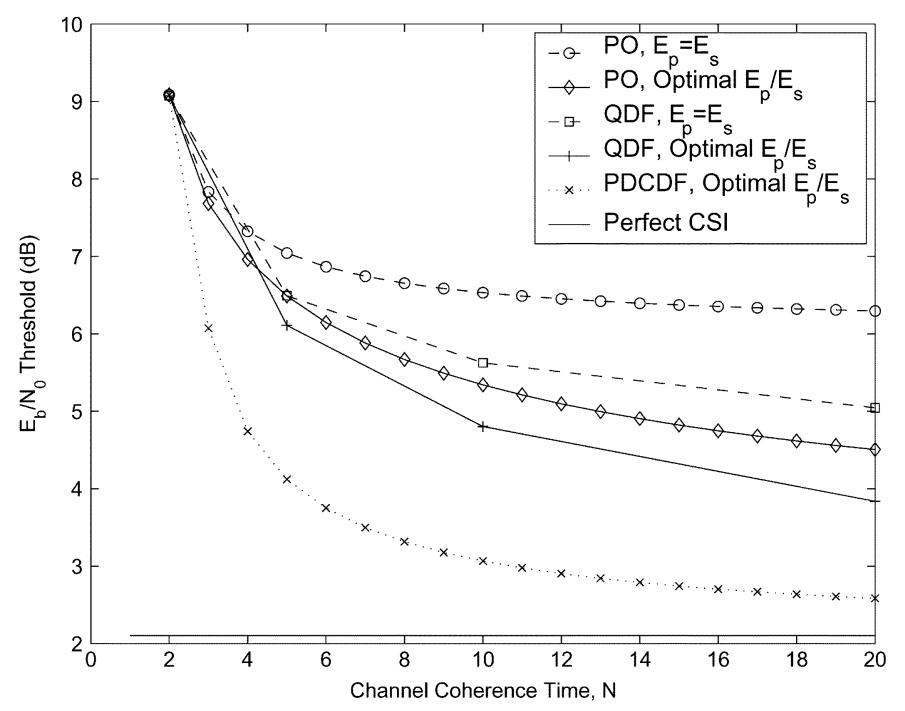

Fig. 3. Discretized density evolution results for the irregular LDPC code of Table I over a complex Gaussian flat fading channel.

code, the PO and PDCDF performances with optimized energy allocations approach the perfect CSI performance for large $N$. When $E_{p} / E_{s}$ is optimized, a performance gain of about $1.2 \mathrm{~dB}$ for the $\mathrm{PO}$ receiver and about $0.8 \mathrm{~dB}$ for the QDF receiver is achieved over the $E_{p}=E_{s}$ case at $N=10$. By using the irregular LDPC code, the $E_{b} / N_{0}$ threshold is about $0.9 \mathrm{~dB}$ lower in the PO case and about $0.6 \mathrm{~dB}$ lower in the QDF case compared to the regular $(3,6) \mathrm{LDPC}$ code at $N=10$ when $E_{p} / E_{s}$ is optimized.

Fig. 3 shows that the PO receiver with optimal $E_{p} / E_{s}$ values performs better than the QDF receiver with $E_{p}=E_{s}$. Thus, simply optimizing the energy allocation in the PO receiver can be more beneficial than using the more complex QDF receiver. When $E_{p} / E_{s}$ is also optimized for the QDF receiver, the gain of the QDF receiver over the PO receiver is $0.5 \mathrm{~dB}$ at $N=10$, which is comparable to the corresponding gain seen in [6] for the noncoherent AWGN channel. When $E_{p}=E_{s}$, the QDF receiver has a more significant benefit over the $\mathrm{PO}$ receiver, i.e., $0.9 \mathrm{~dB}$ at $N=10$ and $1.3 \mathrm{~dB}$ at $N=20$. Comparing this result to the corresponding gains in [11] for turbo codes with a Jake's complex fading channel model, the benefit of iterative decoding and estimation over separate estimation and decoding is approximately $1 \mathrm{~dB}$ smaller here. This can be attributed to several causes: 1) the QDF algorithm is an ad-hoc algorithm which is not a very efficient one for joint iterative detection/estimation, and 2) in the Jake's fading channel model, the fading varies continuously, so greater performance gain can be achieved by utilizing more symbols (in addition to the pilot symbols) for channel estimation than in the block independent model, where the fading is independent from block to block. More precisely, the channel estimation filter in [11] uses 61 symbols whereas the QDF receiver can only use at most $N-1$ symbols (when all bits become biased after several iterations) in the channel estimation.

By optimizing an irregular LDPC code with maximal degrees $\left(d_{v}, d_{c}\right)=(10,8)$ for the QDF receiver at $N=10$ following
TABLE II

Rate 1/2 IRREgular LDPC DegRee Polynomials Optimized FOR THE QDF RECEIVER AT $N=10$

\begin{tabular}{ll|ll}
\hline \hline \multicolumn{2}{c|}{$\lambda(x)$} & \multicolumn{2}{c}{$\rho(x)$} \\
\hline$\lambda_{1}$ & 0.000000 & $\rho_{1}$ & 0.000000 \\
$\lambda_{2}$ & 0.373027 & $\rho_{2}$ & 0.003367 \\
$\lambda_{3}$ & 0.304234 & $\rho_{3}$ & 0.026410 \\
$\lambda_{4}$ & 0.066596 & $\rho_{4}$ & 0.138425 \\
$\lambda_{5}$ & 0.082820 & $\rho_{5}$ & 0.164885 \\
$\lambda_{6}$ & 0.003623 & $\rho_{6}$ & 0.149070 \\
$\lambda_{7}$ & 0.009525 & $\rho_{7}$ & 0.117388 \\
$\lambda_{8}$ & 0.000279 & $\rho_{8}$ & 0.400455 \\
$\lambda_{9}$ & 0.032068 & & \\
$\lambda_{10}$ & 0.127828 & & \\
\hline \hline
\end{tabular}

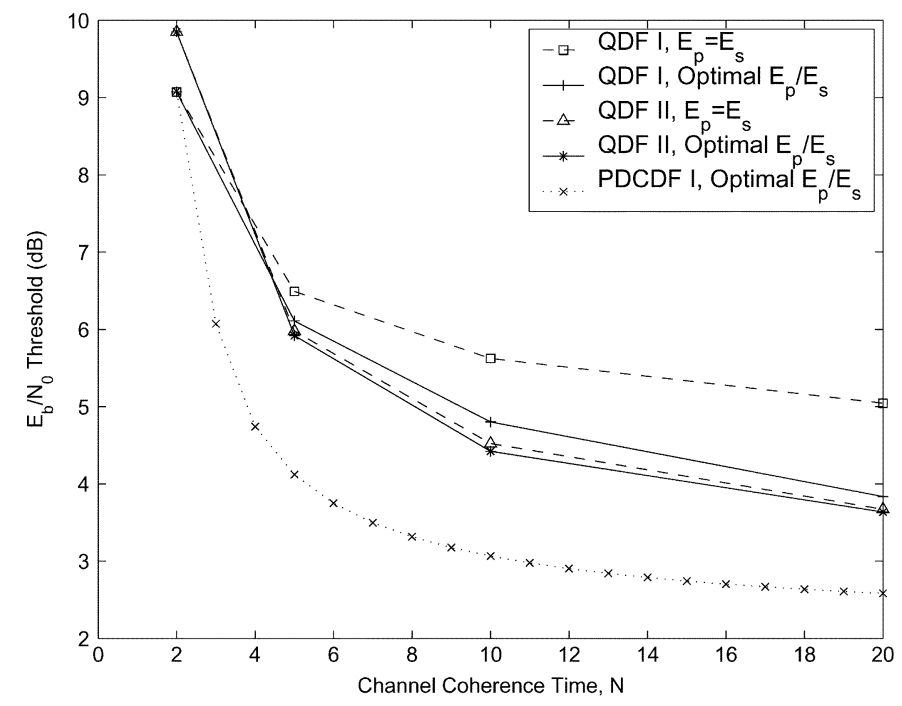

Fig. 4. Performance comparison of the irregular LDPC codes of Tables I and II, denoted by the labels "I" and "II," respectively.

the procedure in Section IV.b, we obtain the code in Table II and this code results in some improvement in performance as shown in Fig. 4. A significant gain of $1.1 \mathrm{~dB}$ is achieved when $E_{p}=E_{s}$ while a small gain of $0.4 \mathrm{~dB}$ is achieved when $E_{p} / E_{s}$ is optimized. Thus, if adjusting $E_{p} / E_{s}$ is a viable option in the transmitter architecture, then not much performance is lost by simply using the code optimized for the perfect CSI receiver.

The optimal energy distributions $E_{p} / E_{s}$ are shown in Fig. 5. The QDF receiver requires less pilot energy than the PO receiver since the coded symbols can also contribute to the channel estimate. For the PO receiver, as $N$ increases, more energy can be used for the pilot symbol to obtain a better channel estimate since the $E_{b} / N_{0}$ penalty due to pilots is reduced as $N$ increases. The previous statement is also true for the QDF receiver when $N$ is small. However, for large $N$ in the QDF receiver, more coded symbols can contribute to the channel estimate, hence, 


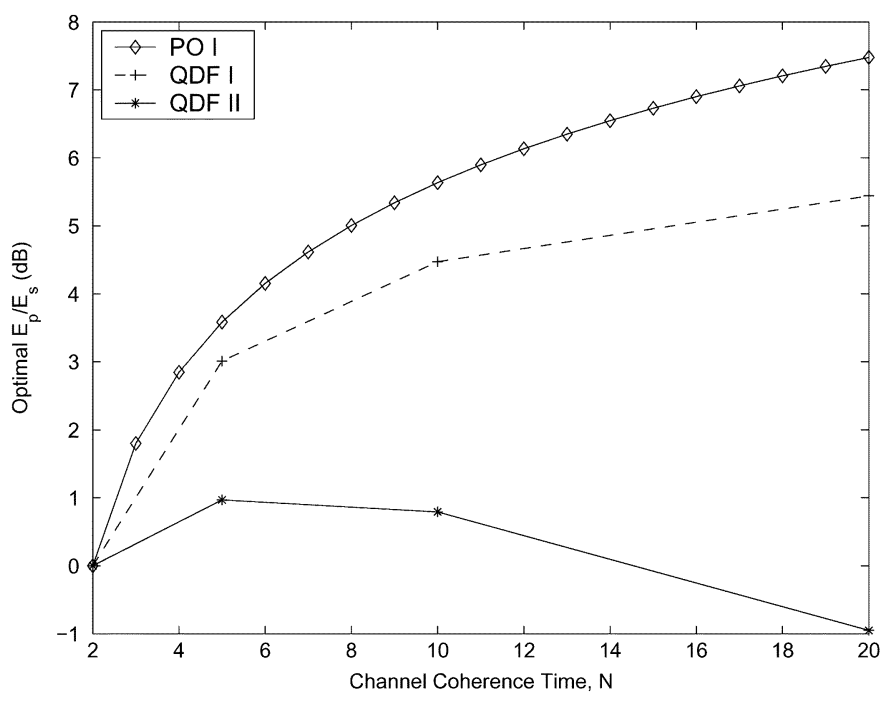

Fig. 5. Optimal $E_{p} / E_{s}$ for PO and QDF receivers for the irregular LDPC codes of Tables I and II, denoted by the labels "I" and "II," respectively.

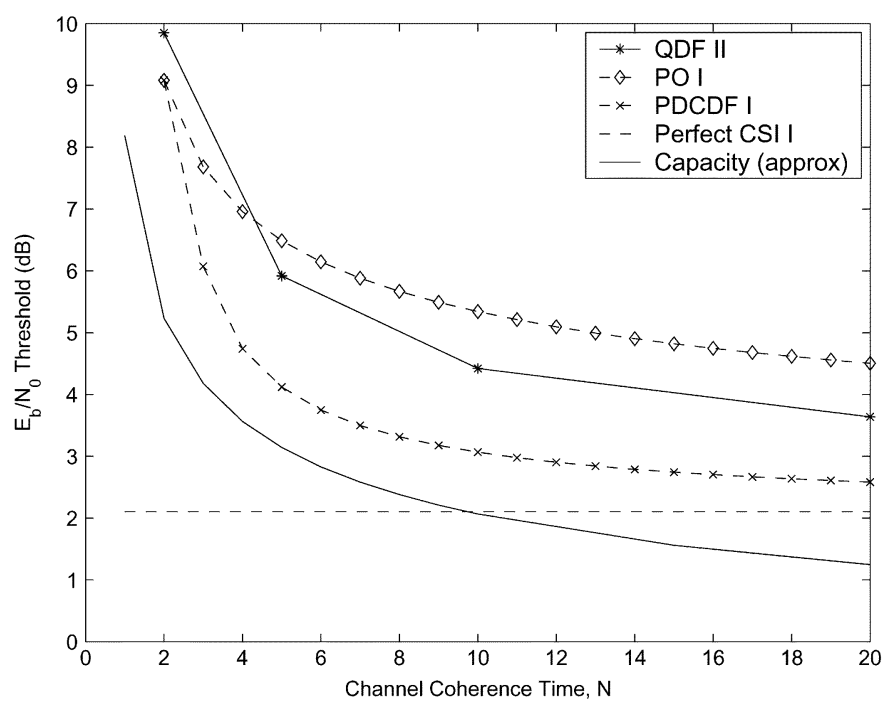

Fig. 6. Performance of the optimized PSA LDPC codes compared to an approximation of the capacity for the complex Gaussian flat fading channel. The labels "I" and "II" denote the irregular LDPC codes in Tables I and II, respectively.

less energy is needed in the pilot symbol. ${ }^{9}$ Although the performance of the QDF receiver with optimized energy allocations is similar for the codes in Tables I and II, the energy allocation that achieves this performance is quite different, as evidenced by the two corresponding curves in Fig. 5. If the transmitter circuitry does not allow for $E_{p} / E_{s}$ values much different than 1 (due to peak power and/or power-added efficiency constraints), then it is beneficial to use the code in Table II which was optimized for the QDF receiver. Thus, for the QDF receiver, good performance can be achieved by optimizing the energy distribution $E_{p} / E_{s}$ and/or by optimizing the LDPC code.

Fig. 6 compares the best performance of the optimized PSA LDPC codes for the perfect CSI, PO, PDCDF, and QDF receivers, where optimization has been completed over the LDPC

${ }^{9}$ This latter trend is also observed in the QDF I curve in Fig. 5 for larger values of $N$ than depicted.

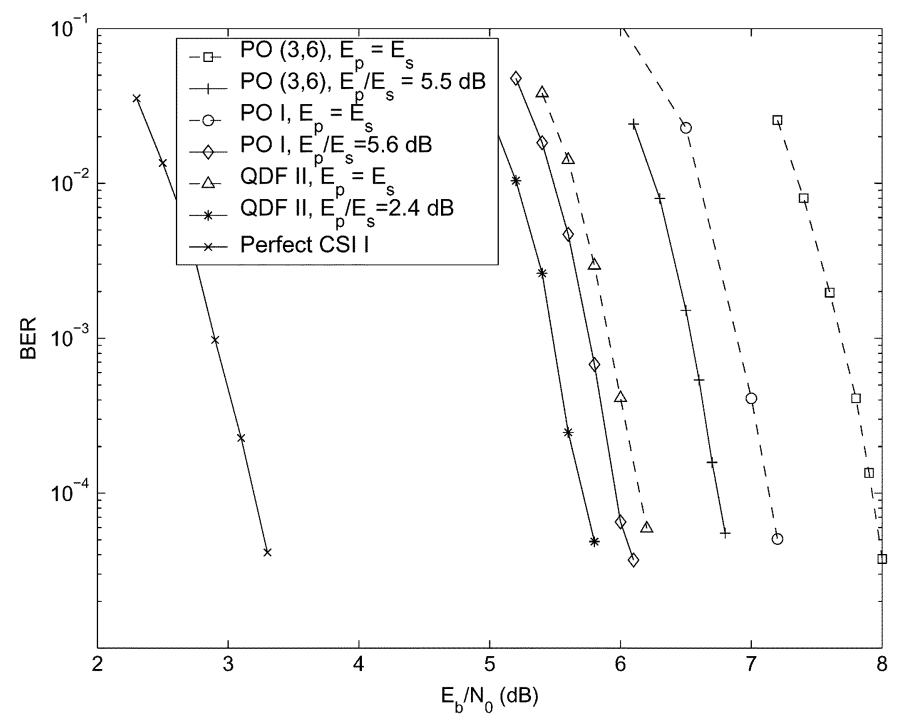

Fig. 7. Simulations of a regular $(3,6)$ LDPC code and the irregular LDPC codes of Table I and Table II, denoted by the labels " $(3,6)$," "I," and "II," respectively, with a codelength of 10008 , channel coherence time of 10, QPSK modulation, and 100 decoding iterations.

code degree polynomials and the energy distribution in the PSA scheme, to an approximation of the capacity for the complex fading channel. The approximation for capacity is obtained by using a two-point approximation with capacity-achieving isotropically distributed inputs [18].

Since the code in Table I is an optimal code for the PDCDF receiver, the PDCDF curve provides a lower bound on the performance of any LDPC code with maximal degrees $\left(d_{v}, d_{c}\right)=$ $(10,8)$ in a PSA scheme with BPSK modulation. Thus, Fig. 6 shows that the performance of such LDPC codes must be at least $1 \mathrm{~dB}$ from capacity.

The best QDF performance provides an improvement of $0.9 \mathrm{~dB}$ over the best PO performance at $N=10$. However, the best QDF performance is still 1.4 dB away from the PDCDF bound and $2.4 \mathrm{~dB}$ away from capacity at $N=10$. The gap between the optimized QDF receiver and capacity may be due to limitations of the PSA scheme, the suboptimality of the QDF receiver, and/or the specific modulation scheme (i.e., BPSK) utilized.

Simulations were conducted to verify the density evolution results and to further investigate the performance of a more practical system utilizing QPSK modulation. For the QDF simulations, a QPSK symbol is used as an effective pilot symbol in the message generation at the channel constraint node only if $\left|r_{i}\right|>T$ for both the LDPC code bits that make up the QPSK symbol. The simulation results for this system are presented in Fig. 7. The simulations were conducted for a regular $(3,6)$ LDPC code and for the rate $1 / 2$ irregular LDPC codes of Tables I and Table II with a codelength of 10008, channel coherence time $N=10, N_{p}=1$, and 100 iterations. For the QDF receiver, the threshold $T$ was set to a fixed value for all iterations and this fixed value was numerically optimized for each simulation point resulting in values of $T$ between 1 and 2.5. The $E_{p} / E_{s}$ values used in Fig. 7 were roughly optimized through simulations for the QPSK modulation scheme. Compared to the 
optimal $E_{p} / E_{s}$ values obtained through density evolution analysis with BPSK modulation, the optimal values here are roughly the same for the PO receiver and 1.4 dB larger for the QDF receiver.

The simulation results show that the proposed iterative algorithms with QPSK modulation have performance roughly comparable to the simulated performance with BPSK modulation. For the PO receiver, performance improves by $0.1-0.2$ $\mathrm{dB}$ with QPSK modulation over BPSK modulation since the penalty due to pilot insertion per information bit is smaller for QPSK modulation, and thus a larger pilot can be used for the same $E_{b} / N_{0}$, resulting in a better channel estimation and better performance. For the QDF receiver, performance degrades by $0.3-0.7 \mathrm{~dB}$ when QPSK modulation is used due in part to the more stringent requirement that both bits have to be biased in order for the QPSK symbol to act as a pilot. With QPSK modulation, the best PO performance is only slightly worse $(0.2 \mathrm{~dB})$ than the performance of the optimized QDF receiver, whereas with BPSK modulation, the optimized QDF receiver provides a $0.9-\mathrm{dB}$ improvement over the optimized $\mathrm{PO}$ receiver in analysis and simulation. Based on this observation, one can argue that well designed PO schemes present an excellent performance/complexity tradeoff, compared to designs based on the QDF and other iterative decoding/estimation techniques.

At this point, a comparison with the performance of receivers in [19] is in order. In [19], turbo codes, convolutional codes, and RA codes in conjunction with differential QPSK modulation are used over a block complex fading channel. The channel estimation portion of the iterative channel estimation/decoding algorithm is completed through an averaging estimator for the fading amplitude and trellis decoding with quantized phases for the fading phase. Simulation results for the QDF receiver, with optimized rate 1/4 LDPC codes, 100 iterations, and all other conditions equivalent to [19], results in about $0.4-0.9 \mathrm{~dB}$ worse performance for $N=10,20$, and 50 compared to the best results reported in [19]. This loss is due largely to the suboptimality of the QDF receiver. However, the complexity of the QDF receiver is much lower than that of the receivers in [19] where the channel estimation requires calculations with 20 quantization levels for the phase and four possibilities for the transmitted QPSK symbol, for each of the $N$ sections in the trellis. Thus, the QDF receiver provides a low-complexity solution at a cost of about $0.4-0.9 \mathrm{~dB}$ in performance.

\section{CONCLUSION}

In this paper, we analyzed and designed PSA LDPC codes over frequency-nonselective, time-selective fading channels where the fading affects both the amplitude and phase of the transmitted symbols and the channel dynamics are taken explicitly into account. Several PSA receivers, including two practical receivers (the PO and QDF receivers) were investigated and analyzed using density evolution.

The performance of the perfect CSI, PO, and PDCDF receivers were shown to be dependent only on a single parameter, $b$. This result led to several important consequences simplifying and unifying the analysis and code design for these receivers and for all system and channel parameters. First, only one set of an- alyzes based on $b$ is necessary to characterize the performance of all three receiver types at any channel coherence time and for any energy allocation. Second, optimizing the energy distribution between pilots and coded symbols only requires a simple closed-form calculation from $b$. Third, an optimal code for one of these receivers will also be optimal for the other receivers and for any system parameters.

Finally, density evolution and simulation results were presented for irregular LDPC codes in a PSA scheme. The allocation of power to the pilots and the coded symbols was optimized resulting in 1-2-dB improvement in performance. By utilizing the iterative joint decoding/estimation provided by the QDF algorithm, the performance improves over the best PO receiver by $0.9 \mathrm{~dB}$ with BPSK modulation and $0.2 \mathrm{~dB}$ with QPSK modulation. Thus, optimized PO schemes for QPSK modulation present an excellent performance/complexity tradeoff. We note however, that a $2.5-\mathrm{dB}$ gap still exists between the performance of the best proposed scheme (optimized QDF receiver) and capacity.

\section{APPENDIX A PROOF OF MONOTONICITY}

The proof of monotonicity of receiver performance, for the perfect CSI, PO, and PDCDF receivers, with the unifying parameter $b$ is given in this Appendix. We will show that if $b_{1}<b_{2}$, then the performance of the receiver characterized by $b=b_{2}$, through the initial density given in (15), is degraded from the receiver characterized by $b=b_{1}$.

For each value of $b$ (regardless of receiver type), there exists an equivalent fading channel with a perfect CSI receiver. This equivalent channel is described by the following expression:

$$
z=c(-1)^{a}+n
$$

where $z$ is the channel output, $(-1)^{a}$ is the channel input where $a \in\{0,1\}, c$ is a zero-mean unit energy complex normal fading coefficient (known at the receiver), and $n$ is a zero-mean complex normal additive noise with energy $N_{0}$ where $N_{0}$ satisfies the equation $b=\left(\sqrt{1+N_{0}}-1\right) / 2$. It can be shown that the $\log$-likelihood ratio for $a$ given by

$$
x=\log \frac{f(z \mid a=0, c)}{f(z \mid a=1, c)}=\frac{4}{N_{0}} \Re\left\{z c^{*}\right\}
$$

is a sufficient statistic for detection in this channel, and that the conditional distribution of $x$ is given by

$$
f(x \mid a=0)=f(-x \mid a=1)=h(x)
$$

where $h(x)$ has the form of (15) with $b=\left(\sqrt{1+N_{0}}-1\right) / 2$.

For $b=b_{1}$, choose $N_{0}$ such that $b_{1}=\left(\sqrt{1+N_{0}}-1\right) / 2$. Since $b_{2}>b_{1}$, we can find $N_{0}^{\prime}=N_{0}+Q>N_{0}$ such that $b_{2}=\left(\sqrt{1+Q+N_{0}}-1\right) / 2$. Based on the above equivalence, this channel corresponds to a perfect CSI fading channel where now more additive noise is injected, i.e.,

$$
z^{\prime}=c(-1)^{a}+n^{\prime}=c(-1)^{a}+n+q=z+q
$$

where the noise component $n^{\prime}$ has energy $N_{0}^{\prime}=Q+N_{0}>$ $N_{0}$ and the log-likelihood sufficient statistic is given by $y=$ 
$4 / N_{0}^{\prime} \Re\left\{z^{\prime} c^{*}\right\}$. The above expression (28) shows that this latter channel is a degraded version of the former, i.e., the output $z$ is further degraded by an additional noise $q$ with variance $Q$. Thus, according to [1, Theorem 1], the performance of any receiver characterized by $b_{2}$ is worse than the performance of any receiver characterized by $b_{1}$.

\section{REFERENCES}

[1] T. J. Richardson and R. L. Urbanke, "The capacity of low-density paritycheck codes under message-passing decoding," IEEE Trans. Inf. Theory, vol. 47, no. 2, pp. 599-618, Feb. 2001.

[2] T. J. Richardson, M. A. Shokrollahi, and R. L. Urbanke, "Design of capacity-approaching irregular low-density parity-check codes," IEEE Trans. Inf. Theory, vol. 47, no. 2, pp. 619-637, Feb. 2001.

[3] J. Hou, P. H. Siegel, and L. B. Milstein, "Performance analysis and code optimization of low-density parity-check codes on raleigh fading channels," IEEE J. Sel. Areas Commun., vol. 19, no. 3, pp. 924-934, May 2001

[4] R. J. McEliece, "Are turbo-like codes effective on nonstandard channels?," in IEEE Inf. Theory Soc. Newslett., vol. 51, Dec. 2001, pp. 1-8.

[5] H. Jin and T. J. Richardson, "Design of low-density parity-check codes for noncoherent MPSK communication," in Proc. Int. Symp. Information Theory, Lausanne, Switzerland, Jun. 2002, p. 169.

[6] R. Nuriyev and A. Anastasopoulos, "Pilot-symbol-assisted coded transmission over the block-noncoherent AWGN channel," IEEE Trans. Commun., vol. 51, no. 6, pp. 953-963, Jun. 2003.

[7] T. L. Marzetta and B. M. Hochwald, "Capacity of a mobile multipleantenna communication link in Rayleigh flat fading," IEEE Trans. Inf. Theory, vol. 45, no. 1, pp. 139-157, Jan. 1999.

[8] B. Hassibi and B. Hochwald, "How much training is needed in multipleantenna wireless links?," IEEE Trans. Inf. Theory, vol. 49, no. 4, pp. 951-963, Apr. 2003.

[9] L. Zheng and D. N. C. Tse, "Communication on the Grassmann manifold: A geometric approach to the noncoherent multiple-antenna channel," IEEE Trans. Inf. Theory, vol. 48, no. 2, pp. 359-383, Feb. 2002.

[10] A. Anastasopoulos and K. M. Chugg, "Adaptive soft-input soft-output algorithms for iterative detection with parametric uncertainty," IEEE Trans. Commun., vol. 48, no. 10, pp. 1638-1649, Oct. 2000.

[11] M. C. Valenti and B. D. Woerner, "Iterative channel estimation and decoding of pilot symbol assisted turbo codes over flat-fading channels," IEEE J. Sel. Areas Commun., vol. 19, no. 9, pp. 1697-1705, Sep. 2001.

[12] R. Storn and K. Price, "Differential evolution-A simple and efficient heuristic for global optimization over continuous spaces," J. Global Optimization, vol. 11, pp. 341-359, 1997.

[13] B. J. Frey, Graphical Models for Machine Learning and Digital Communications. Cambridge, MA: MIT Press, 1998.

[14] A. P. Worthen and W. E. Stark, "Unified design of iterative receivers using factor graphs," IEEE Trans. Inf. Theory, vol. 47, no. 2, pp. 843-849, Feb. 2001.
[15] J. G. Proakis, Digital Communications, 4th ed. New York: McGrawHill, 2001.

[16] K. Fu and A. Anastasopoulos, "Performance analysis of LDPC codes for time-selective complex fading channels," in Proc. Globecom Conf. Taipei, Taiwan, R.O.C., Nov. 2002, pp. 1279-1283.

[17] S.-Y. Chung, G. D. Forney Jr., T. J. Richardson, and R. L. Urbanke, "On the design of of low-density parity-check codes within $0.0045 \mathrm{~dB}$ of the Shannon limit," IEEE Commun. Lett., vol. 5, no. 2, pp. 58-60, Feb. 2001.

[18] I. C. A. Faycal, M. D. Trott, and S. Shamai (Shitz), "The capacity of discrete-time rayleigh fading channels," in Proc. Int. Symp. Information Theory, Ulm, Germany, Jun. 1997, p. 473.

[19] R.-R. Chen, R. Koetter, U. Madhow, and D. Agrawal, "Joint noncoherent demodulation and decoding for the block fading channel: A practical framework for approaching Shannon capacity," IEEE Trans. Commun., vol. 51, no. 10, pp. 1676-1689, Oct. 2003.

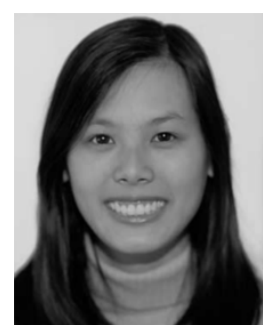

Kaiann Fu (S'02) received the B.S.E. and M.S.E. degrees in electrical engineering from the University of Michigan, Ann Arbor, in 2000 and 2001, respectively. She is currently pursuing the Ph.D. degree at the University of Michigan and is a National Science Foundation Graduate Research Fellowship recipient

Her research interests include coding theory, LDPC codes, iterative algorithms, and coding for fading channels.

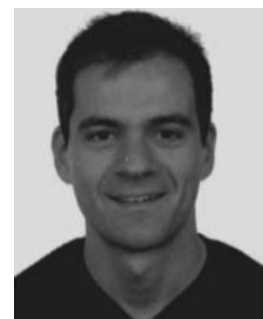

Achilleas Anastasopoulos (S'97-M'99) was born in Athens, Greece, in 1971. He received the Diploma degree in electrical engineering from the National Technical University of Athens, Athens, Greece, in 1993, and the M.S. and Ph.D. degrees in electrical engineering from the University of Southern California, Los Angeles, in 1994 and 1999, respectively.

$\mathrm{He}$ is currently an Assistant Professor in the Department of Electrical Engineering and Computer Science, University of Michigan, Ann Arbor. His research interests lie in the general area of communication theory, with emphasis in joint parameter estimation and data detection, adaptive iterative algorithms, and coding for fading channels. He is the co-author of the book Iterative Detection: Adaptivity, Complexity Reduction, and Applications, (Reading, MA: Kluwer Academic, 2001).

Dr. Anastasopoulos is the recipient of the "Myronis Fellowship" in 1996 from the Graduate School at the University of Southern California, and the NSF CAREER Award in 2004. He served as a Technical Program Committee Member for ICC 2003 and Globecom 2004. He currently serves on the Editorial Board of the IEEE TRANSACTIONS ON COMMUNICATIONS 